\title{
3D numerical simulations of propagating two-fluid, torsional Alfvén waves and heating of a partially-ionized solar chromosphere
}

\author{
B. Kuźma, ${ }^{1,2 \star}$ K. Murawski, ${ }^{2}$ and S. Poedts ${ }^{1,2}$ \\ ${ }^{1}$ Center for Mathematical Plasma Astrophysics, Department of Mathematics, KU Leuven, Celestijnenlaan 200B, 3001 Leuven \\ ${ }^{2}$ Institute of Physics, University of Maria Curie-Skłodowska, Pl. M. Curie-Skłodowskiej 5, 20-031 Lublin, Poland
}

Accepted XXX. Received YYY; in original form ZZZ

\begin{abstract}
We present a new insight into the propagation, attenuation and dissipation of two-fluid, torsional Alfvén waves in the context of heating of the lower solar atmosphere. By means of numerical simulations of the partially-ionized plasma, we solve the set of two-fluid equations for ion plus electron and neutral fluids in three-dimensional (3D) Cartesian geometry. We implement initially a current-free magnetic field configuration, corresponding to a magnetic flux-tube that is rooted in the solar photosphere and expands into the chromosphere and corona. We put the lower boundary of our simulation region in the low chromosphere, where ions and neutrals begin to decouple, and implement there a monochromatic driver that directly generates Alfvén waves with a wave period of $30 \mathrm{~s}$. As the ion-neutral drift increases with height, the two-fluid effects become more significant and the energy carried by both Alfvén and magneto-acoustic waves can be thermalized in the process of ion-neutral collisions there. In fact, we observe a significant increase in plasma temperature along the magnetic flux-tube. In conclusion, the two-fluid torsional Alfvén waves can potentially play a role in the heating of the solar chromosphere.
\end{abstract}

Key words: Sun: activity - Sun: photosphere - Sun: chromosphere - Sun: transition region - methods: numerical

\section{INTRODUCTION}

To understand the multitude of physical processes occurring on the Sun, one has to know how the solar plasma dynamics is determined by the solar magnetic field. One of the omnipresent magnetic structures in the solar photosphere are magnetic flux-tubes. Observed usually in the photosphere and low chromosphere (e.g., Hansteen et al. 2006; De Pontieu et al. 2007; Rouppe van der Voort et al. 2009; Kuridze et al. 2015; Srivastava et al. 2017) and rooted in between granules, they are prone to highly turbulent motions associated with the solar granulation. The latter is an efficient source of waves that are propagating into the solar atmosphere, and some of which were interpreted as magnetohydrodynamic-gravity waves (e.g., Musielak \&

^ E-mail: blazej.kuzma@kuleuven.be
Ulmschneider 2001). Recent high-resolution observations revealed that the solar atmosphere is indeed permeated by diversity of waves, which in some cases carries energy-flux sufficient to heat the atmospheric plasma (e.g., Jess et al. 2009; De Pontieu et al. 2014; Morton et al. 2015; Jafarzadeh et al. 2017; Srivastava et al. 2017). In the light of these discoveries, wave heating is widely discussed as a potential solution of the coronal heating problem. The energy transport from the photosphere, through the chromosphere and into the corona strongly depends on the efficiency of wave reflection from the inhomogeneous regions (Hollweg et al. 1982; Cranmer \& van Ballegooijen 2005). In particular, the mentioned omnipresent solar magnetic flux-tubes may act as guidelines for Alfvén waves, which were theoretically predicted by Alfvén (1942), and have been potentially observed in the solar atmosphere (e.g., Tomczyk et al. 
2007; Srivastava et al. 2017). The linear Alfvén waves propagation in the isothermal solar atmosphere was investigated numerically and analytically and cut-off frequencies were revealed by Perera et al. (2015). It was also shown that a finely-structured flux-tube can be a guide not only for Alfvén waves, but also for fast magneto-acoustic kink waves (Kuźma \& Murawski 2018) and localized Alfvén pulses may play potential role in vertical mass transport and formation of lower solar atmospheric jets (Scalisi et al. 2021). However, the actual mechanisms of energy dissipation, and thus its efficiency and ability to compensate the radiative losses, is still a matter of ongoing discussion.

The many different types of waves have complex interactions: mode-coupling can transfer energy from one mode into another, the waves refract while propagating through the inhomogeneous atmosphere, also their polarization changes as they propagate, and real physical absorption and conversion of the wave power into heat can take place on top of all of those other processes (Khomenko et al. 2018). Neutrals can possibly play an important role in photosphere and chromosphere, as plasma is only partially ionized there (e.g., Zaqarashvili et al. 2011). The collisions between ions and neutrals can lead, among other things, to wave damping and energy dissipation (e.g., Piddington 1956; Watanabe 1961; Kulsrud \& Pearce 1969; Haerendel 1992; De Pontieu \& Haerendel 1998; James \& Erdélyi 2002; Erdélyi \& James 2004; Khodachenko et al. 2004; Forteza et al. 2007). It was demonstrated that also the properties of Alfvén waves are prone to partial ionization (Soler et al. 2013). The above mentioned collisional energy dissipation and as a result collisional heating should be taken into consideration in the context of the ongoing discussion on heating of the chromosphere. Thus, the two-fluid processes are essential for a correct description of the wave-processes occurring in the lower layers of the solar atmosphere.

The chromospheric heating by Alfvén waves due to two-fluid effects, mainly ion-neutral collisions, was first discussed in the pioneering paper of Piddington (1956) who considered waves propagating in a partially-ionised gas with ionized plasma and neutral gas treated as separated, independently moving fluids. He estimated the frictional heating in the chromosphere as $10^{5} \mathrm{erg} \mathrm{cm}^{-2} \mathrm{~s}^{-1}$. Later, Osterbrock (1961) argued this value to be overestimated, pointing out that the effective cross-section for ion-neutral collisions is larger and the real frequency spectrum is considerably lower and the frictional damping-heating rate is in fact very small. Haerendel (1992) discussed weakly damped Alfvén waves, due to ion-neutral collisions, as drivers of solar chromospheric spicules. Goodman (2011) investigated the effect of Pedersen current dissipation of driven in the photosphere Alfvén oscillations on the chromospheric heating rate. He showed that the latter is comparable with the observationally estimated radiative losses of $10^{7} \mathrm{erg} \mathrm{cm}^{-2} \mathrm{~s}^{-1}$ in the chromosphere. Zaqarashvili et al. (2013), using a single-fluid magnetohydrodynamic (MHD) model supplemented by a Cowling diffusion coefficient in the presence of neutral helium, showed strong damping of short-period torsional Alfvén waves by ion-neutral collisions. The propagation of Alfvén waves, and their collisional dissipation and heating in a 1.5D partially ionised solar chromosphere, was studied by De Pontieu et al. (2001), Leake et al. (2005), and Tu \& Song (2013). Additionally, Arber et al. (2016) using a 1.5D non-ideal MHD modelling, demonstrated that it is possible to heat the chromospheric plasma by direct resistive dissipation of high-frequency Alfvén waves through Pedersen resistivity. Shelyag et al. (2016) modelled non-linear wave propagation in a magnetic flux tube with the effect of partial ionization taken into account by the ambipolar term from the generalized Ohm's law in a single-fluid quasi-MHD regime. They showed, that ambipolar diffusion can lead to effective dissipation of MHD waves perturbation and, as result, to temperature increase in chromospheric magnetic structures. Soler et al. (2017), and Soler et al. (2019) investigated energy transport and heating by torsional Alfvén waves propagating along magnetic flux-tubes expanding from the photosphere to the low corona, with the assumption of stationary state propagation. They found that waves with wave periods shorter than $20 \mathrm{~s}$ are dissipated in the chromosphere, and thereby the energy sufficient to balance the radiative losses is thermalized there. They also demonstrated that in the photosphere heating through Ohmic dissipation is dominating over heating through ion-neutral collisions.

For a long time it was a subject of intensive discussions whether two-fluid effects play any role in the low frequency regime (e.g., Soler et al. 2013; Ballai 2019, among others). Recently published numerical results strongly suggest that ion-neutral collisions can affect the transport of energy and its dissipation in a partially-ionized chromosphere. For instance, Soler et al. (2019), using a photospheric broadband driver that contains a spectrum of frequencies corresponding to wave periods ranging from $3.33 \mathrm{~s}$ up to $2.78 \mathrm{~h}$, investigated plasma heating and energy transfer by two-fluid Alfvén waves. Popescu Braileanu et al. (2019) showed collisional damping 
and energy dissipation for monochromatic waves with wave periods starting from $1 \mathrm{~s}$ up to $20 \mathrm{~s}$. Moreover, in the recent works on linear and nonlinear two-fluid Alfvén waves Martínez-Gómez et al. (2018) and Kuźma et al. (2020) revealed a rise of the plasma temperature in the solar atmosphere resulting from the collisional heating. The two-fluid effects also play an important role when non-linear effects become important, particularly in the context of wave damping and plasma heating (e.g., Kuźma et al. 2019; Wójcik et al. 2020; Zhang et al. 2021). On top of that, Murawski et al. (2020) demonstrated that ion-neutral collisions may solely solve the heating problem of a quiet region of the chromosphere.

The main goal of the present paper is to investigate the impact of two-fluid effects on (a) propagation, attenuation and dissipation of torsional Alfvén waves, (b) chromospheric heating, and (c) energy transfer to the upper layers of the solar corona. We aim to follow and complement both the previous studies of Popescu Braileanu et al. (2019) and Kuźma et al. (2019) by taking into account a 3D model of the solar atmosphere, and the study of Soler et al. (2019) by taking into account a non-static, time-varying model of propagating Alfvén waves. As we want to focus solely on two-fluid effects on wave propagation and plasma heating, we limited our model by not taking into account non-ideal terms in the induction equation, nor ionization and recombination (Popescu Braileanu et al. 2019) and resistivity (Soler et al. 2019). Ultimately, we want to determine and stress the importance of two-fluid effects for processes with time-scales longer than the characteristic time-scales of ion-neutral collisions.

This paper is organized as follows. In Section 2, we present the two-fluid equations for ions plus electrons and neutrals. In Section 3 , we describe the 3D magneto-hydrostatic model of a partially-ionized solar atmosphere and present results of numerical simulations. Finally, we conclude and summarize our work in Section 4.

\section{TWO-FLUID PLASMA MODEL}

To model the lower atmospheric layers of the Sun, we consider a gravitationally stratified and magnetically confined plasma that consists of two components: electric charges (ions plus electrons) and neutral fluid (neutrals) that interact via ion-neutral collisions. The non-ideal terms in the induction equation, ionization and recombination (Popescu Braileanu et al. 2019) as well as resistivity (Soler et al. 2019) are not considered in the present model. This model is governed by the equations describing mass conservation:

$$
\begin{aligned}
& \frac{\partial \varrho_{\mathrm{n}}}{\partial t}+\nabla \cdot\left(\varrho_{\mathrm{n}} \mathbf{V}_{\mathrm{n}}\right)=0, \\
& \frac{\partial \varrho_{\mathrm{i}}}{\partial t}+\nabla \cdot\left(\varrho_{\mathrm{i}} \mathbf{V}_{\mathrm{i}}\right)=0 ;
\end{aligned}
$$

the momentum equations:

$\varrho_{\mathrm{n}} \frac{\partial \mathbf{V}_{\mathrm{n}}}{\partial t}+\varrho_{\mathrm{n}}\left(\mathbf{V}_{\mathrm{n}} \cdot \nabla\right) \mathbf{V}_{\mathrm{n}}=-\nabla p_{\mathrm{n}}+\varrho_{\mathrm{n}} \mathbf{g}-\alpha_{\mathrm{c}}\left(\mathbf{V}_{\mathrm{n}}-\mathbf{V}_{\mathrm{i}}\right)$,

$\varrho_{\mathrm{i}} \frac{\partial \mathbf{V}_{\mathrm{i}}}{\partial t}+\varrho_{\mathrm{i}}\left(\mathbf{V}_{\mathrm{i}} \cdot \nabla\right) \mathbf{V}_{\mathrm{i}}=$

$-\nabla p_{\mathrm{i}}+\frac{1}{\mu}(\nabla \times \mathbf{B}) \times \mathbf{B}+\varrho_{\mathrm{i}} \mathbf{g}+\alpha_{\mathrm{c}}\left(\mathbf{V}_{\mathrm{n}}-\mathbf{V}_{\mathrm{i}}\right) ;$

and the energy equations:

$\frac{\partial E_{\mathrm{n}}}{\partial t}+\nabla \cdot\left(\left(E_{\mathrm{n}}+p_{\mathrm{n}}\right) \mathbf{V}_{\mathrm{n}}\right)=Q_{\mathrm{n}}+\left(\varrho_{\mathrm{n}} \mathbf{g}-\alpha_{\mathrm{c}}\left(\mathbf{V}_{\mathrm{n}}-\mathbf{V}_{\mathrm{i}}\right)\right) \cdot \mathbf{V}_{\mathrm{n}}$,

$\frac{\partial E_{\mathrm{i}}}{\partial t}+\nabla \cdot\left[\left(E_{\mathrm{i}}+p_{\mathrm{i}}+\frac{\mathbf{B}^{2}}{2 \mu}\right) \mathbf{V}_{\mathrm{i}}-\frac{\mathbf{B}}{\mu}\left(\mathbf{V}_{\mathrm{i}} \cdot \mathbf{B}\right)\right]=$

$Q_{\mathrm{i}}+\left(\varrho_{\mathrm{i}} \mathbf{g}+\alpha_{\mathrm{c}}\left(\mathbf{V}_{\mathrm{n}}-\mathbf{V}_{\mathrm{i}}\right)\right) \cdot \mathbf{V}_{\mathrm{i}}$

which are supplemented by induction equation and the solenoidal condition:

$\frac{\partial \mathbf{B}}{\partial t}=\nabla \times\left(\mathbf{V}_{\mathrm{i}} \times \mathbf{B}\right), \quad \nabla \cdot \mathbf{B}=0$.

Here,

$E_{\mathrm{n}}=\frac{p_{\mathrm{n}}}{\gamma-1}+\frac{1}{2} \varrho_{n}\left|\mathbf{V}_{\mathrm{n}}\right|^{2}$,

and

$E_{\mathrm{i}}=\frac{p_{\mathrm{i}}}{\gamma-1}+\frac{1}{2} \varrho_{\mathrm{i}}\left|\mathbf{V}_{\mathrm{i}}\right|^{2}+\frac{|\mathbf{B}|^{2}}{2 \mu}$,

where subscripts $\mathrm{n}$ and $\mathrm{i}_{\mathrm{i}}$ denotes ion and neutral components of energy densities, $E_{\mathrm{n}}\left(E_{\mathrm{i}}\right)$, mass densities, $\varrho_{\mathrm{n}}\left(\varrho_{\mathrm{i}}\right)$, and temperatures, $T_{\mathrm{n}}\left(T_{\mathrm{i}}\right)$. The gas pressure is indicated for neutrals by $p_{\mathrm{n}}$, and for ions plus electrons by $p_{\mathrm{i}}$. The constant $\mu$ is the magnetic permeability of the medium, $\mathbf{B}$ denotes the magnetic field, and $\gamma=5 / 3$ the adiabatic index. The gravitational acceleration vector is directed towards negative $y$-axis in the Cartesian coordinate system we consider and its magnitude is $g=274.78 \mathrm{~m} \mathrm{~s}^{-2}$.

Note, that in our model we consider hydrogen as the main plasma component. The influence from heavier elements is taken from the OPAL solar abundances repository (e.g., Vögler et al. 2005). The mean masses of ions plus electrons and neutrals are $\mu_{\mathrm{i}}=0.58$ and $\mu_{\mathrm{n}}=1.21$, respectively. The above-described fluids interact via ion-neutral collisions. The effect of the interaction between these 
fluids depends on the ion-neutral friction coefficient. Following Zaqarashvili et al. (2011), we assume that $\alpha_{\mathrm{in}}=\alpha_{\mathrm{ni}}=\alpha_{\mathrm{c}}$, and with use of formula derived by Braginskii (1965) we find:

$\alpha_{\mathrm{c}}=\frac{4}{3} \frac{\sigma_{\mathrm{in}} \varrho_{\mathrm{i}} \varrho_{\mathrm{n}}}{m_{H}\left(\mu_{\mathrm{i}}+\mu_{\mathrm{n}}\right)} \sqrt{\frac{8 k_{B}}{\pi m_{H}}\left(\frac{T_{\mathrm{i}}}{\mu_{\mathrm{i}}}+\frac{T_{\mathrm{n}}}{\mu_{\mathrm{n}}}\right)}$.

Here, $m_{\mathrm{H}}$ corresponds to the hydrogen mass, $k_{B}$ is Boltzmann's constant, and $\sigma_{\text {in }}$ is the ion-neutral collision cross-section taken as a quantum value (Vranjes \& Krstic 2013). Note, that the frictional interaction between these fluids results in additional heat production and exchange terms proportional, respectively, to the square of the velocity difference and the temperature difference (e.g., Ballester et al. 2018, and references cited therein):

$Q_{\mathrm{n}}=\alpha_{c}\left[\frac{1}{2}\left|\mathbf{V}_{\mathrm{i}}-\mathbf{V}_{\mathrm{n}}\right|^{2}-\frac{3}{2} \frac{k_{B}}{m_{\mathrm{H}}\left(\mu_{\mathrm{i}}+\mu_{\mathrm{n}}\right)}\left(T_{\mathrm{n}}-T_{\mathrm{i}}\right)\right]$,
$Q_{\mathrm{i}}=\alpha_{c}\left[\frac{1}{2}\left|\mathbf{V}_{\mathrm{i}}-\mathbf{V}_{\mathrm{n}}\right|^{2}-\frac{3}{2} \frac{k_{B}}{m_{\mathrm{H}}\left(\mu_{\mathrm{i}}+\mu_{\mathrm{n}}\right)}\left(T_{\mathrm{i}}-T_{\mathrm{n}}\right)\right]$.

This frictional heating is a two-fluid effect, however it can be studied in single-fluid MHD models that include the ambipolar term in the induction equation and the corresponding ambipolar heating term in the energy equation when the two fluids are strongly coupled. In this case, the frictional heating term consistently reverts to the ambipolar heating term in the single-fluid approximation (Khomenko et al. 2014; Ballester et al. 2018). We study its impact on partially-ionized plasma behaviour and contribution to overall heating within a twofluid model. From Eqs. (11)-(12) we infer that collisional heating arises when the mass densities of both fluids are similar and both fluids are still coupled (thus non-zero $\alpha_{\mathrm{c}}$ ), but this coupling is weak enough to allow ion-neutral drift, $\mathbf{V}_{\mathrm{i}}-\mathbf{V}_{\mathrm{n}}$, attaining non-zero values. We infer that these conditions are met in the chromosphere.

\section{NUMERICAL SIMULATIONS OF 3D ALFVÉN WAVES}

\subsection{Hydrostatic equilibrium model of the solar atmosphere}

In our model, the solar atmosphere is assumed to be initially (at $t=0 \mathrm{~s})$ in a hydrostatic $\left(\mathbf{V}_{\mathrm{i}, \mathrm{n}}=\mathbf{0}\right)$ equilibrium. Taking the ideal gas law for ion and neutral fluids into account:

$p_{\mathrm{n}}=\frac{k_{\mathrm{B}}}{m_{\mathrm{H}} \mu_{\mathrm{n}}} \varrho_{\mathrm{n}} T_{\mathrm{n}}, \quad$ and $\quad p_{\mathrm{i}}=\frac{k_{\mathrm{B}}}{m_{\mathrm{H}} \mu_{\mathrm{i}}} \varrho_{\mathrm{i}} T_{\mathrm{i}}$,
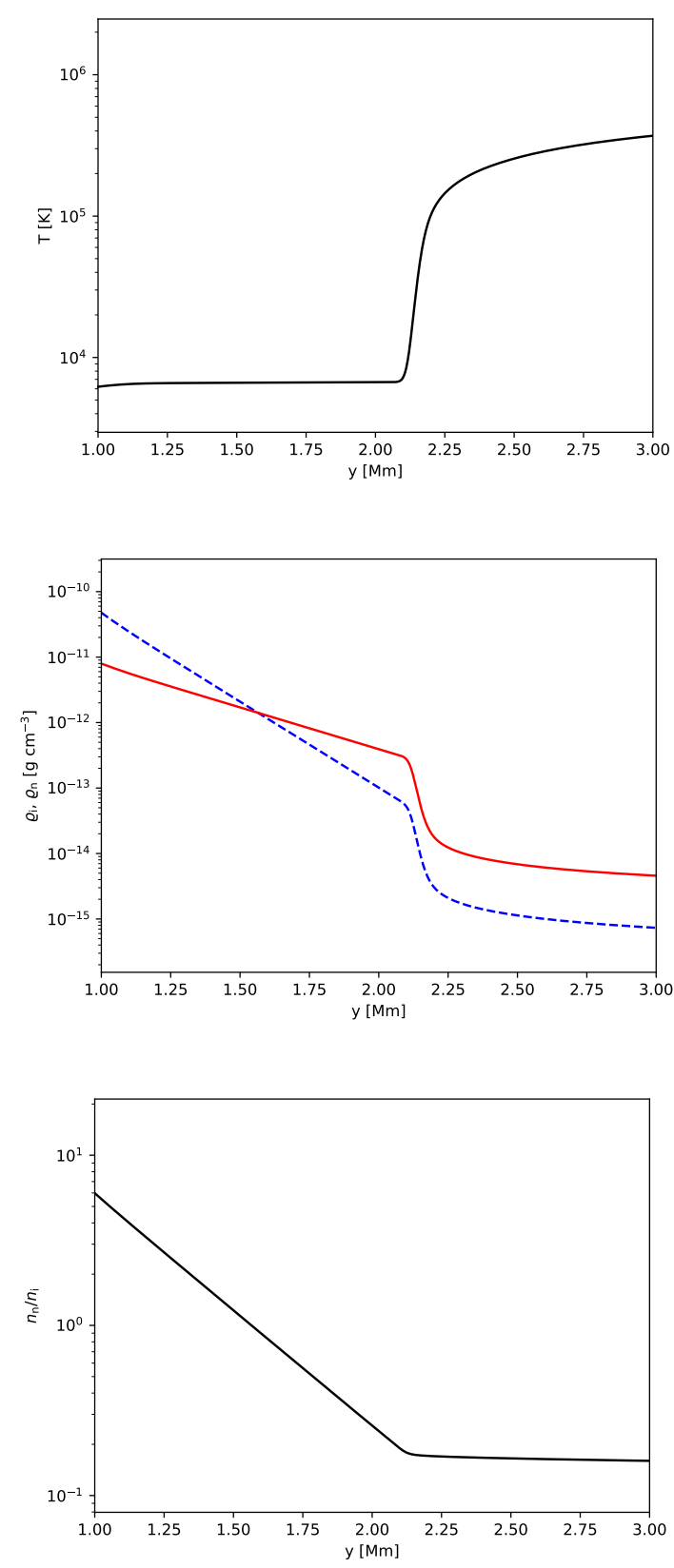

Figure 1. Vertical profiles of temperature $T$ (top), corresponding ion $\varrho_{\mathrm{i}}$ (solid line, middle) and neutral $\varrho_{\mathrm{n}}$ (dashed line, middle) mass densities, and the ionization ratio $n_{\mathrm{n}} / n_{\mathrm{i}}$ (bottom).

and from the $y$-components of hydrostatic $\left(-\nabla p_{\mathrm{i}, \mathrm{n}}+\varrho_{\mathrm{i}, \mathrm{n}} \mathbf{g}=\mathbf{0}\right)$ equations, we derive the equilibrium gas pressures:

$$
\begin{aligned}
& p_{\mathrm{n}}(y)=p_{0 \mathrm{n}} \exp \left[-\int_{y_{\mathrm{ref}}}^{y} \frac{d y}{\Lambda_{\mathrm{n}}(y)}\right], \\
& p_{\mathrm{i}}(y)=p_{0 \mathrm{i}} \exp \left[-\int_{y_{\mathrm{ref}}}^{y} \frac{d y}{\Lambda_{\mathrm{i}}(y)}\right] .
\end{aligned}
$$

Here, $p_{0 \mathrm{n}}=3 \cdot 10^{-3}$ dyn $\mathrm{cm}^{-2}\left(p_{0 \mathrm{i}}=10^{-1} \mathrm{dyn}^{-2}\right)$ corresponds to the pressure of neutrals (ions) at the reference level, $y_{\text {ref }}=50 \mathrm{Mm}$, 


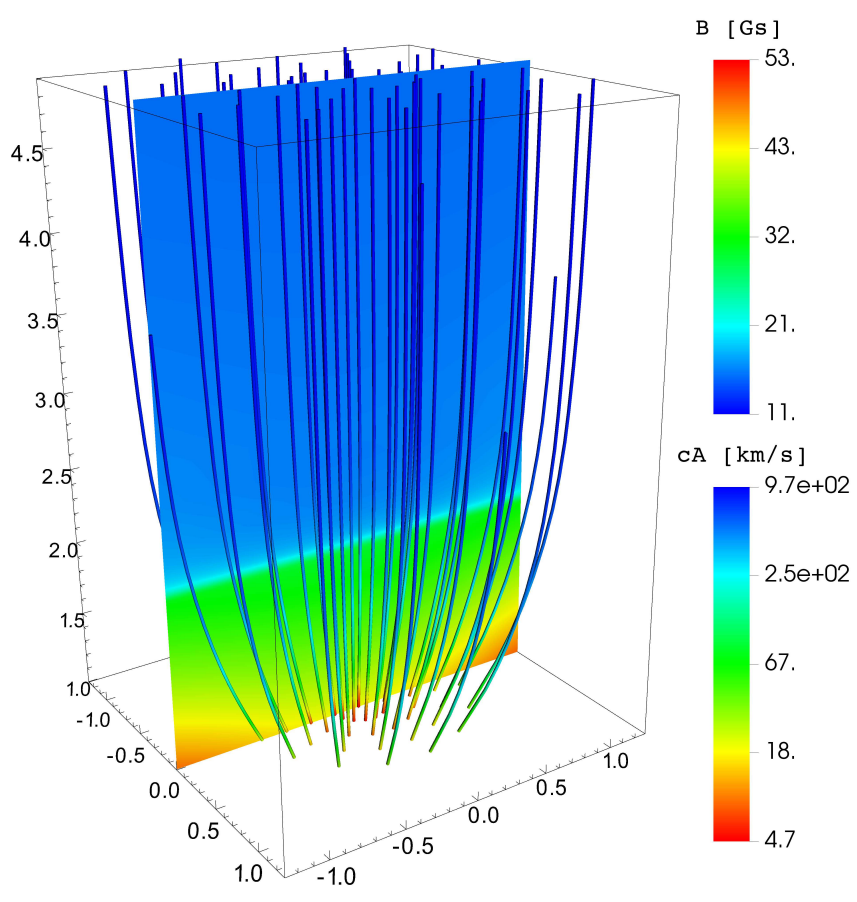

Figure 2. The equilibrium magnetic field lines with magnetic field strength, $B$, expressed in Gauss and the equilibrium Alfvén speed profile, $c_{A}$, expressed in $\mathrm{km} \mathrm{s}^{-1}$.

and

$\Lambda_{\mathrm{n}}=\frac{k_{\mathrm{B}} T(y)}{m_{\mathrm{H}} \mu_{\mathrm{n}} g}, \quad$ and $\quad \Lambda_{\mathrm{i}}=\frac{k_{\mathrm{B}} T(y)}{m_{\mathrm{H}} \mu_{\mathrm{i}} g}$,

are, the neutral (ion) pressure scale-heights, respectively. Note that the ion and neutral mass density profiles depend solely on the temperature (Fig. 1, top), which is taken from the semi-empirical model of Avrett \& Loeser (2008). As a result of differences between these profiles (Fig. 1, middle), the ionization degree depends on height above the photosphere. Starting from the weakly ionized bottom of the chromosphere, where the neutral mass density exceeds the ion mass density by about an order of magnitude, through ion-neutral equilibrium at about $900 \mathrm{~km}$ below the located at $y \approx 2.1 \mathrm{Mm}$ transition region, to the one million hot solar corona which, as the mass density of neutrals experiences a sudden fall-off with height, is essentially fully ionized (Fig. 1, bottom).

In the above described equilibrium model, we implement a currentfree, and thus force-free, magnetic field with the configuration of a magnetic flux-tube. In 3D Cartesian coordinates this structure is described as (Low 1985)

$\left[B_{x}, B_{y}, B_{z}\right]=$

$\frac{S\left[-3 x(y-a), x^{2}-2(y-a)^{2}+z^{2},-3(y-a) z\right]}{\left(x^{2}+(y-a)^{2}+z^{2}\right)^{5 / 2}}$

$+\left[0, B_{\mathrm{V}}, 0\right]$

where the magnetic field singularity is located at $y=a$, the free parameter $S$ determines its strength and $B_{\mathrm{V}}=10 \mathrm{G}$ is additional vertical component of magnetic field. As a result, the described magnetic structure mimics a chromospheric magnetic flux-tube with strength of its magnetic field equals to $B=53 \mathrm{G}$ at $y=1 \mathrm{Mm}$ above the photosphere, extending up into the corona, and attaining a magnetic field strength of $B=10 \mathrm{G}$ at height of $y=5 \mathrm{Mm}$, with magnetic lines becoming essentially vertical at this level (Fig. 2, solid lines). When extrapolated to the photosphere, the investigated flux-tube will be rooted in-between the granules with a magnetic field strength of $B=850 \mathrm{G}$ at its foot-point. The spatial profile of the corresponding Alfvén speed, $c_{\mathrm{A}}(x, y)=B / \sqrt{\mu\left(\varrho_{\mathrm{i}}+\varrho_{\mathrm{n}}\right)}$, is illustrated in Fig. 2 (colormap). In the lower chromosphere $c_{\mathrm{A}}$ attains values lower than $10 \mathrm{~km} \mathrm{~s}^{-1}$ outside the flux-tube, while in its center, given by $x=z=0 \mathrm{Mm}$ it attains value of $20 \mathrm{~km} \mathrm{~s}^{-1}$. As a result of decreasing mass density it rises higher up exceeding value of $50 \mathrm{~km}$ $\mathrm{s}^{-1}$ and experiencing sudden jump to $900 \mathrm{~km} \mathrm{~s}^{-1}$ at the transition region.

\subsection{Numerical box and perturbations}

To solve the two-fluid equations numerically, we use the JOANNA code (Wójcik et al. 2018; Wójcik et al. 2019). For the considered problem, we set the Courant-Friedrichs-Lewy number equal to 0.3 and choose the TVDLF approximate Riemann solver (Toro 2009). We set the simulation box in $(x, y, z)$ as $(-1.28,1.28) \mathrm{Mm} \times(1,30) \mathrm{Mm}$ $\times(-1.28,1.28) \mathrm{Mm}$, where $y=1 \mathrm{Mm}$ corresponds to $500 \mathrm{~km}$ above the photosphere. In the numerical simulations, we use a uniform grid within the region below the level given by $y=3.56 \mathrm{Mm}$. This region is covered by $256 \times 256 \times 256$ grid points, which leads to a spatial resolution of $10 \mathrm{~km}$ there. Above this region, i.e. in the corona, we implement a divided into 128 cells stretched vertically grid whose cells sizes grow with increasing $y$. As a result of that, any upward propagating signal becomes strongly diffused in the upper layers. This allows us to avoid significant reflections from the upper boundary. At the top, bottom, and sides, we set all the plasma quantities equal to their equilibrium values, given by Eqs. (14)-(17) and $\mathbf{V}_{\mathrm{i}, \mathrm{n}}=\mathbf{0}$. 

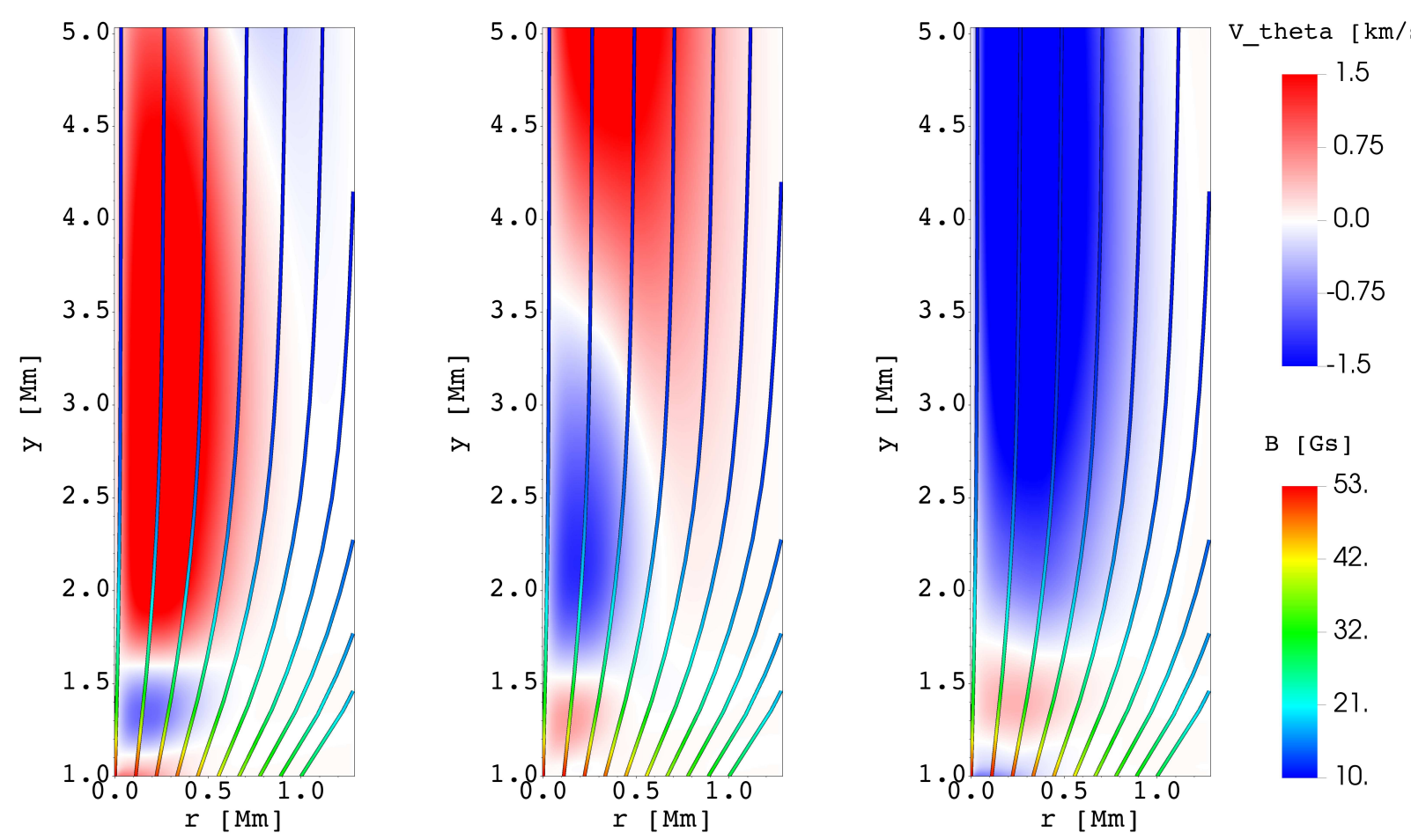

Figure 3. Three spatial profiles of the azimuthal ion velocity, $V_{\mathrm{i} \theta}(r, y)$, with $r=\sqrt{x^{2}+z^{2}}$, at $t=980 \mathrm{~s}, t=990 \mathrm{~s}$, and $t=1000 \mathrm{~s}$ (from left to right) overlayed by magnetic field lines, the case of $P_{\mathrm{d}}=30 \mathrm{~s}$ and $A_{V}=1 \mathrm{~km} \mathrm{~s}^{-1}$.

To excite the torsional Alfvén waves at the bottom boundary, we set the periodic azimuthal driver in ion and neutral velocities, which written in Cartesian coordinates takes the form

$\left[V_{\mathrm{i} x}, V_{\mathrm{i} y}, V_{\mathrm{i} z}\right]=\left[V_{\mathrm{n} x}, V_{\mathrm{n} y}, V_{\mathrm{n} z}\right]=$

$A_{V}[-z, 0, x] \exp \left(-\frac{x^{2}+z^{2}}{w^{2}}\right) \sin \left(\frac{2 \pi}{P_{\mathrm{d}}} t\right)$,

where $A_{V}=1 \mathrm{~km} \mathrm{~s}^{-1}$ is the effective amplitude, $w=0.1 \mathrm{Mm}$ the width and $P_{\mathrm{d}}$ the period of the driver which we set equal to $30 \mathrm{~s}$.

\subsection{Numerical results}

Figure 3 shows the spatial profiles of azimuthal ion velocity component, $V_{\mathrm{i} \theta}(r, y, t)$, with $r=\sqrt{x^{2}+z^{2}}$, at three instants of time, namely at $t=980 \mathrm{~s}, t=990 \mathrm{~s}$ and $t=1000 \mathrm{~s}$ (from left to right) for $P_{\mathrm{d}}=30 \mathrm{~s}, A_{V}=1 \mathrm{~km} \mathrm{~s}^{-1}$, and $w=100 \mathrm{~km}$. The torsional driver excites Alfvén waves that propagates upwardly along the expanding solar magnetic-flux tube. The amplitude of these waves grows exponentially with the distance at a rate that equals to the pressure-scale height (e.g., Kuźma \& Murawski 2018), however this effect is reduced by collisional damping. This fact has a two-fold effect on the overall picture. First, less energy is thermalized at the lower layers, and second, more energy can be transferred to the upper layers and contribute to the plasma heating there. The pressure scale-height amplitude growth becomes dominant in the upper chromosphere and above the transition region. At the lower chromospheric heights, the ion-neutral coupling is already weak enough for neutrals to depart from the evolution of the ions. It was shown in previous studies on two-fluid acoustic waves (Kuźma et al. 2019) that even in nonmagnetic case both ion and neutral fluids start to decouple in the chromosphere. Here, the magnetic field is an additional factor amplifying this effect. The dynamic motions of ions are directly altered by the presence of magnetic field. Obviously, the latter has no such influence on neutrals and, as result, they get extra freedom to propagate in the radial direction. The ion-neutral drift increases, and, as it 

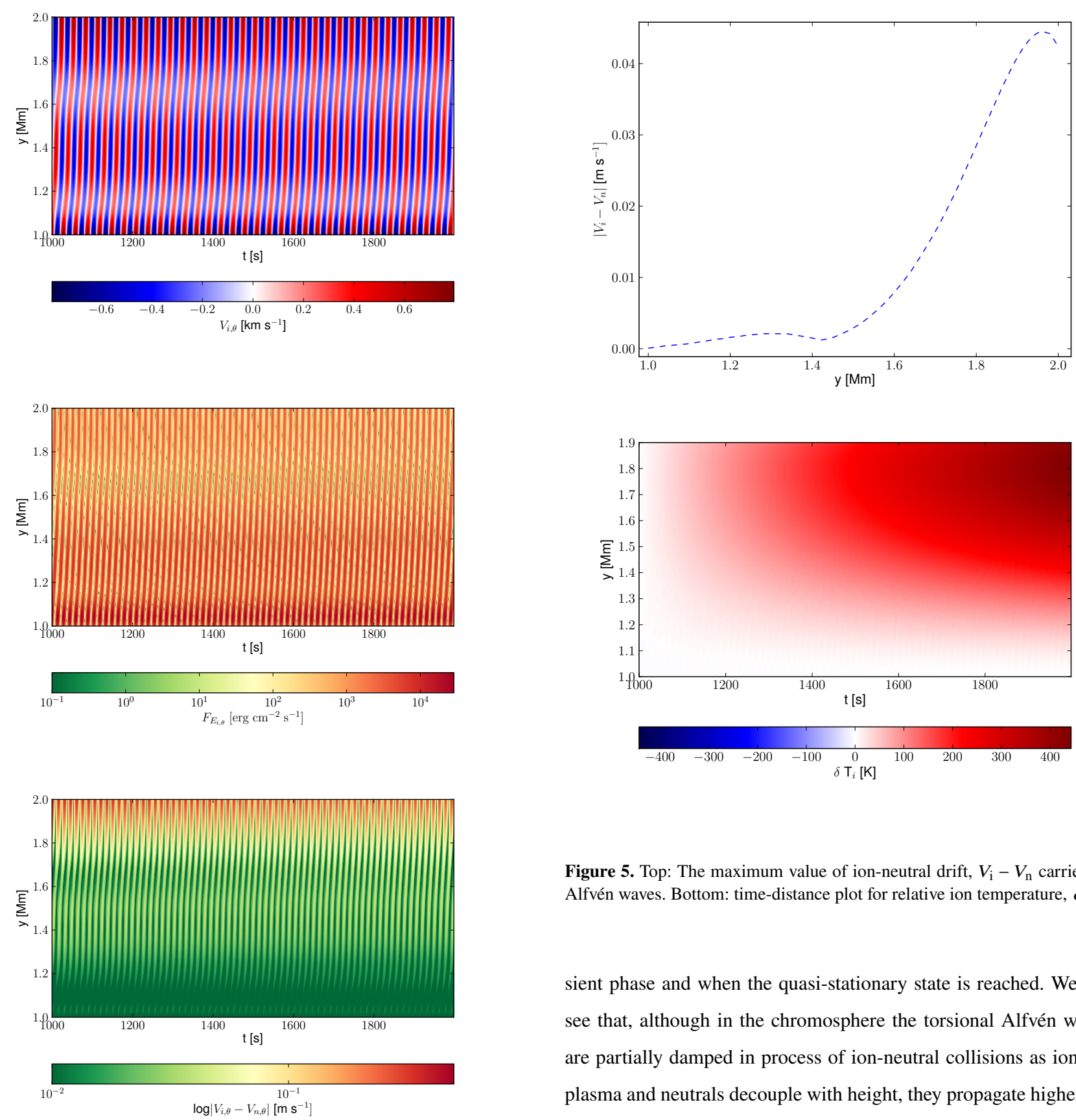

Figure 4. Time-distance plots for azimuthal components of ion velocity, $V_{\mathrm{i} \theta}(r=0.1 \mathrm{Mm})$, ion energy flux, $F_{E_{\mathrm{i}} \theta}(r=0.1 \mathrm{Mm})$, and ion-neutral drift, $\log \left|V_{\mathrm{i} \theta}-V_{\mathrm{n} \theta}\right|(r=0.1 \mathrm{Mm})$, from top to bottom.

will be shown later, starts playing a significant role in the process of plasma heating according to Eqs. (11)-(12).

The top panel of Fig. 4 illustrates a time-distance plot of the azimuthal component of the ion velocity collected $100 \mathrm{~km}$ out of the flux-tube center, $V_{\mathrm{i} \theta}(r=0.1 \mathrm{Mm})$. Note that only part of the timeline for $t>10^{3} \mathrm{~s}$ is displayed, namely the period after the tran-

Figure 5. Top: The maximum value of ion-neutral drift, $V_{\mathrm{i}}-V_{\mathrm{n}}$ carried by Alfvén waves. Bottom: time-distance plot for relative ion temperature, $\delta T_{\mathrm{i}}$.

sient phase and when the quasi-stationary state is reached. We can see that, although in the chromosphere the torsional Alfvén waves are partially damped in process of ion-neutral collisions as ionized plasma and neutrals decouple with height, they propagate higher up, ultimately reaching the transition region and corona above (compare with Fig. 3). This stays in an agreement with recent findings of Soler et al. (2019). The transverse MHD waves (i.e., Alfvén and kink waves) takes part in the process of transport of the energy into and through the upper layers of the solar atmosphere. This energy, if thermalized, can play a significant role in the heating of the chromosphere and corona (e.g., Dwivedi \& Srivastava 2010; Sokolov et al. 2013; Murawski \& Musielak 2016; Srivastava et al. 2017). Note that even small ion-neutral drift multiplied by friction coefficient, $\alpha_{c}$, may result in noticeable frictional heating (Eqs. 11 - 12), and thus ion-neutral collisions may play a role in thermalizing energy carried 


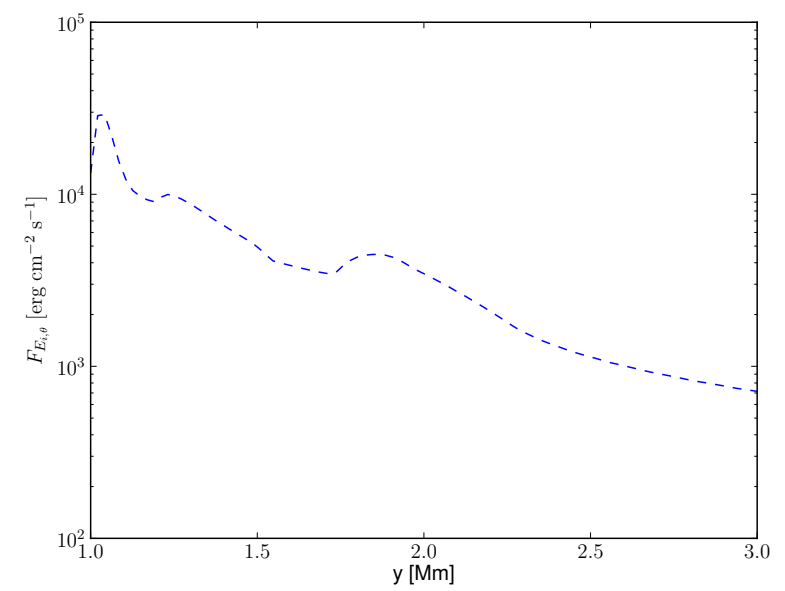

Figure 6. The maximum value of ion energy flux, $F_{E_{i}}$ carried by Alfvén waves versus height, $y$.

by Alfvén waves. The middle panel of Fig. 4 illustrates the corresponding time-distance plot of the Alfvén waves ion energy flux, described by the following formula (Mathioudakis et al. 2013):

$F_{E_{\mathrm{i}, \theta}}=\varrho_{\mathrm{i}} c_{\mathrm{A}} V_{\mathrm{i}, \theta}$.

As we can see, the torsional Alfvén waves carry along the flux-tube an energy flux of about $10^{4} \mathrm{erg} \mathrm{cm}^{-2} \mathrm{~s}^{-1}$. This value falls off with height to about $10^{3} \mathrm{erg} \mathrm{cm}^{-2} \mathrm{~s}^{-1}$ right above the transition region due to abrupt drop in ion density, $\varrho_{\mathrm{i}}$ (not shown).

As we discussed in Section 2, one of the possible mechanism of dissipation of the carried energy is frictional heating resulting from ion-neutral collisions. The bottom panel of Fig. 4 illustrates the ionneutral drift, $\log \left|V_{\mathrm{i}}-V_{\mathrm{n}}\right|(r=0.1 \mathrm{Mm})$ with the $-\theta$ component taken into account, thus for Alfvén waves. From Eqs. (11)-(12), we infer that the frictional heating of the plasma by torsional Alfvén waves is dominant in the chromosphere and significantly grows with height. Although Alfvén waves are present in the solar corona, frictional heating cannot contribute effectively to coronal heating due to the low abundance of neutrals, as the plasma becomes essentially fullyionized there.

Figure 5 reveals the maximum value of ion-neutral drift, $\mid V_{\mathrm{i} \theta}-$ $V_{\mathrm{n} \theta} \mid$ versus height, $y$ (top) and time-distance evolution of the relative ion temperature, $\delta T_{\mathrm{i}}(r=0.1 \mathrm{Mm}, t)=T_{\mathrm{i}}(r=0.1 \mathrm{Mm}, t)-T_{0}$ (bottom). The ion-neutral drift associated with Alfvén waves experiences constant growth above the height of $y=1.1 \mathrm{Mm}$ and abruptly jump above $y=1.6 \mathrm{Mm}$. We see that the energy dissipated in the process of frictional heating leads to a significant increase of the

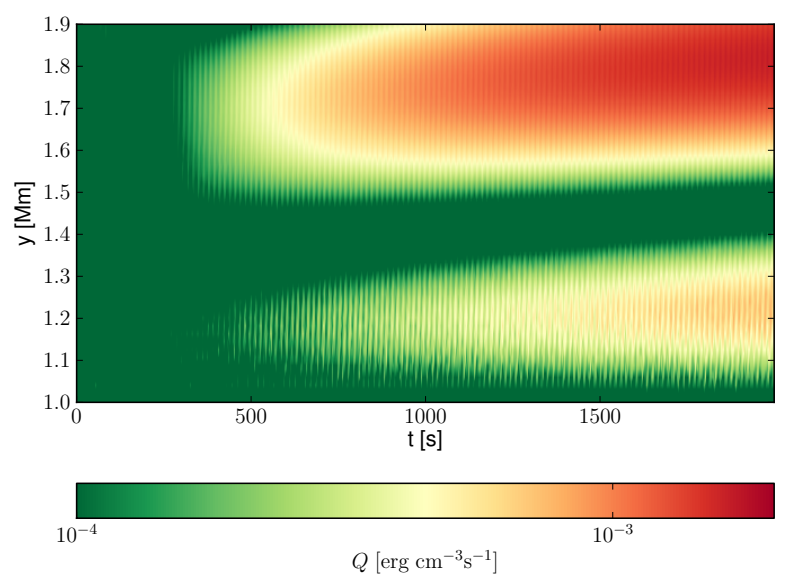

Figure 7. Time-distance plot for the frictional heating rate taken from $Q_{\mathrm{i}}$ term.

plasma temperature, mainly in the upper chromosphere just below the transition region. Note that the relative plasma temperature shares many similarities to the heating pattern of driven in the photosphere acoustic waves (Kuźma et al. 2019) and 2.5D Alfvén waves (Kuźma et al. 2020).

Figure 6 shows the height profile of the maximum ion energy flux carried by Alfvén waves as described by Eq. (19). Note that after the initial transient phase $(t<50 \mathrm{~s})$ this profile remains constant in time and depends solely on the driver amplitude. We see that Alfvén waves in terms of energy transport remain relevant along the entire flux-tube. This energy is partially dissipated in the chromosphere by ion-neutral collisions (compare with Fig. 5, bottom). As a result of dissipation, only $10 \%$ of the initially pumped into system energy reaches the corona as torsional Alfvén waves. Note that these waves may potentially still dissipate their energy in the corona contributing to coronal heating. As we can see, the torsional Alfvén waves carry an energy flux of about $10^{4} \mathrm{erg} \mathrm{cm}^{-2} \mathrm{~s}^{-1}$ in the chromosphere and this value falls off with height to about $10^{3} \mathrm{erg} \mathrm{cm}^{-2} \mathrm{~s}^{-1}$ right above the transition region. In comparison to chromospheric and coronal radiative energy losses for quiet Sun estimated by Withbroe \& Noyes (1977), the total energy flux carried by Alfvén waves is an order of magnitude too small to compensate these losses. Figure 7 illustrates the time-distance plot of collisional heating rate obtained in simulation and taken from Eq. (12). The obtained value of heating rate is also one order of magnitude too small in comparison with the estimated chromospheric cooling rate which in the middle and upper solar chromosphere attains a value of $\sim 10^{-2} \mathrm{erg} \mathrm{cm}^{-3} \mathrm{~s}^{-1}$ (Avrett 
et al. 2015). This scenario may change if we take non-linear Alfvén waves into consideration (Kuźma et al. 2020).

\section{CONCLUSIONS}

By means of numerical simulations we described a partially-ionized solar plasma using two-fluid equations for ions plus electrons and neutral gas, respectively. We coupled these fluids with ion-neutral collisions, and, finally, we generated monochromatic Alfvén waves that originate below the chromosphere. We let them propagate along the current-free magnetic field configuration of a magnetic flux-tube and investigated their attenuation and dissipation. We followed and extended the previous studies of Popescu Braileanu et al. (2019) and Kuźma et al. (2019) by taking into account a 3D model of the solar atmosphere, and the study of Soler et al. (2019) by taking into account non-static, time-varying model of propagating Alfvén waves.

We found that the magnetic flux-tube acts as a guideline for the driven torsional Alfvén waves. These waves propagate upwards into the chromosphere and corona. However, only part of the carried waveenergy is transferred above the transition region. Part of this energy is thermalized in the chromosphere in the process of collisional heating. We found that although the chromospheric plasma temperature rises in time, both the energy carried by two-fluid Alfvén waves and collisional heating can compensate the radiative losses only partially and thus these Alfvén waves alone are not sufficient to explain the chromospheric heating. We also infer that as a result of a dissipation $10 \%$ of the initial energy input reaches the corona in form of Alfvén waves.

Our conclusion has important implications on constructing theoretical models of the solar atmosphere. It was showed that the twofluid effects may play significant, if not crucial role in wave processes in the chromosphere and energy transfer between the photosphere and corona. Therefore they should be included into the model. Perhaps even more important, we showed that two-fluid effects are essential not only for short wave periods. We note that the only dissipation mechanism considered in this work is ion-neutral collisions which, according to our results, do not produce enough heating to compensate radiative losses in the chromosphere. This agrees with previous findings. However, if additional dissipation mechanisms were included, the heating would probably be larger as their associated heating rates are even greater than those of ion-neutral collisions, which has been explored in a number of studies (see, for instance,
Goodman 2011; Tu \& Song 2013; Arber et al. 2016; Soler et al. 2019). In particular, magnetic diffusion has been shown to be the dominant heating mechanism in the most part of the chromosphere (essentially at low and medium altitudes). Further research is required to determine the feasibility of constructing the realistic 3D model, i.e. with thermal conduction, radiative cooling, non-local thermal equilibrium in the chromosphere and magnetic resistivity terms taken into account, sustaining its quasi-equilibrium with two-fluid Alfvén waves heating.

\section{ACKNOWLEDGEMENTS}

The JOANNA code was developed at the Institute of Mathematics of University of M. Curie-Skłodowska, Lublin, Poland by Darek Wójcik. This work was supported as part of project funded by National Science Centre (NCN) grant nos. 2017/25/B/ST9/00506 and 2020/37/B/ST9/00184. SP and BK acknowledges support through the projects C14/19/089 (C1 project Internal Funds KU Leuven), G.0D07.19N (FWO-Vlaanderen), SIDC Data Exploitation (ESA Prodex-12). This project (EUHFORIA 2.0) has received funding from the European Union's Horizon 2020 research and innovation programme under grant agreement No 870405.

\section{DATA AVAILABILITY STATEMENT}

The data underlying this article will be shared on reasonable request to the corresponding author.

\section{REFERENCES}

Alfvén H., 1942, Nature, 150, 405

Arber T. D., Brady C. S., Shelyag S., 2016, ApJ, 817, 94

Avrett E., Loeser R., 2008, ApJS, 175, 229

Avrett E., Tian H., Landi E., Curdt W., Wülser J. P., 2015, ApJ, 811, 87

Ballai I., 2019, Frontiers in Astronomy and Space Sciences, 6, 39

Ballester J. L., et al., 2018, Space Sci. Rev., 214, 58

Braginskii S., 1965, Reviews of Plasma Physics, 1, 205

Cranmer S. R., van Ballegooijen A. A., 2005, ApJS, 156, 265

De Pontieu B., Haerendel G., 1998, A\&A, 338, 729

De Pontieu B., Martens P. C. H., Hudson H. S., 2001, ApJ, 558, 859

De Pontieu B., et al., 2007, Science, 318, 1574

De Pontieu B., et al., 2014, Science, 346, 1255732

Dwivedi B. N., Srivastava A. K., 2010, Current Science, 98, 295 
Erdélyi R., James S. P., 2004, A\&A, 427, 1055

Forteza P., Oliver R., Ballester J. L., Khodachenko M. L., 2007, A\&A, 461, 731

Goodman M. L., 2011, ApJ, 735, 45

Haerendel G., 1992, Nature, 360, 241

Hansteen V. H., De Pontieu B., Rouppe van der Voort L., van Noort M., Carlsson M., 2006, ApJ, 647, L73

Hollweg J. V., Jackson S., Galloway D., 1982, Sol. Phys., 75, 35

Jafarzadeh S., et al., 2017, ApJS, 229, 9

James S. P., Erdélyi R., 2002, Spicule formation by ion-neutral damping. ESA Special Publication Vol. 2

Jess D. B., Mathioudakis M., Erdelyi R., Crockett P. J., Keenan F. P., Christian D. J., 2009, Science, 323, 1582

Khodachenko M. L., Arber T. D., Rucker H. O., Hanslmeier A., 2004, A\&A, 422,1073

Khomenko E., Collados M., Díaz A., Vitas N., 2014, Phys. Plasmas, 21, 092901

Khomenko E., Vitas N., Collados M., de Vicente A., 2018, A\&A, 618, A87

Kulsrud R., Pearce W. P., 1969, ApJ, 156, 445

Kuridze D., Henriques V., Mathioudakis M., Erdélyi R., Zaqarashvili T. V., Shelyag S., Keys P. H., Keenan F. P., 2015, ApJ, 802, 26

Kuźma B., Murawski K., 2018, ApJ, 866, 50

Kuźma B., Wójcik D., Murawski K., 2019, ApJ, 878, 81

Kuźma B., Wójcik D., Murawski K., Yuan D., Poedts S., 2020, A\&A, 639, A45

Leake J. E., Arber T. D., Khodachenko M. L., 2005, A\&A, 442, 1091

Low B. C., 1985, ApJ, 293, 31

Martínez-Gómez D., Soler R., Terradas J., 2018, ApJ, 856, 16

Mathioudakis M., Jess D. B., Erdélyi R., 2013, Space Sci. Rev., 175, 1

Morton R. J., Tomczyk S., Pinto R., 2015, Nature Communications, 6, 7813

Murawski K., Musielak Z. E., 2016, MNRAS, 463, 4433

Murawski K., Musielak Z. E., Wójcik D., 2020, ApJ, 896, L1

Musielak Z. E., Ulmschneider P., 2001, A\&A, 370, 541

Osterbrock D. E., 1961, ApJ, 134, 347

Perera H. K., Musielak Z. E., Murawski K., 2015, MNRAS, 450, 3169

Piddington J. H., 1956, MNRAS, 116, 314

Popescu Braileanu B., Lukin V. S., Khomenko E., de Vicente Á., 2019, A\&A, $627, \mathrm{~A} 25$

Rouppe van der Voort L., Leenaarts J., de Pontieu B., Carlsson M., Vissers G., 2009, ApJ, 705, 272

Scalisi J., Oxley W., Ruderman M. S., Erdélyi R., 2021, ApJ, 911, 39

Shelyag S., Khomenko E., de Vicente A., Przybylski D., 2016, ApJ, 819, L11

Sokolov I. V., et al., 2013, ApJ, 764, 23

Soler R., Carbonell M., Ballester J. L., 2013, ApJS, 209, 16

Soler R., Terradas J., Oliver R., Ballester J. L., 2017, ApJ, 840, 20
Soler R., Terradas J., Oliver R., Ballester J. L., 2019, ApJ, 871, 3

Srivastava A. K., et al., 2017, Scientific Reports, 7, 43147

Tomczyk S., McIntosh S. W., Keil S. L., Judge P. G., Schad T., Seeley D. H., Edmondson J., 2007, Science, 317, 1192

Toro E. F., 2009, Riemann Solvers and Numerical Methods for Fluid Dynamics. Springer Berlin Heidelberg, doi:10.1007/b79761, https:

//doi.org/10.1007/b79761

Tu J., Song P., 2013, ApJ, 777, 53

Vögler A., Shelyag S., Schüssler M., Cattaneo F., Emonet T., Linde T., 2005, A\&A, 429, 335

Vranjes J., Krstic P. S., 2013, A\&A, 554, A22

Watanabe T., 1961, Canadian Journal of Physics, 39, 1197

Withbroe G. L., Noyes R. W., 1977, Annu. Rev. Astron. Astrophys., 15, 363 Wójcik D., Murawski K., Musielak Z. E., 2018, MNRAS

Wójcik D., Kuźma B., Murawski K., Srivastava A. K., 2019, ApJ, 884, 127

Wójcik D., Kuźma B., Murawski K., Musielak Z. E., 2020, A\&A, 635, A28

Zaqarashvili T. V., Khodachenko M. L., Rucker H. O., 2011, A\&A, 529, A82

Zaqarashvili T. V., Khodachenko M. L., Soler R., 2013, A\&A, 549, A113

Zhang F., Poedts S., Lani A., Kuźma B., Murawski K., 2021, ApJ, 911, 119 